\title{
Comprehending the therapeutic effects of stereotactic body radiation therapy for small hepatocellular carcinomas based on imagings
}

\author{
Zhao-Chong Zeng', Jia Fan², Jian Zhou², Meng-Su Zeng ${ }^{3}$, Yi-Xing Chen', Zhi-Feng Wu', Jing Sun', Jian-Ying \\ Zhang', Yong Hu', Qian-Qian Zhao'
}

\begin{abstract}
'Department of Radiation Oncology, Zhongshan Hospital, Fudan University, Shanghai 200032, China.
${ }^{2}$ Liver Cancer Institute, Zhongshan Hospital, Fudan University, Shanghai 200032, China.

${ }^{3}$ Department of Radiology, Zhongshan Hospital, Fudan University, Shanghai 200032, China.
\end{abstract}

Correspondence to: Dr. Zhao-Chong Zeng, Department of Radiation Oncology, Zhongshan Hospital, Fudan University, Shanghai 200032, China. E-mail: zeng.zhaochong@zs-hospital.sh.cn

How to cite this article: Zeng ZC, Fan J, Zhou J, Zeng MS, Chen YX, Wu ZF, Sun J, Zhang JY, Hu Y, Zhao QQ. Comprehending the therapeutic effects of stereotactic body radiation therapy for small hepatocellular carcinomas based on imagings. Hepatoma Res2020;6:43. http://dx.doi.org/10.20517/2394-5079.2020.29

Received: 23 Mar 2020 First Decision: 29 Apr 2020 Revised: 6 May 2020 Accepted: 28 May 2020 Published: 10 Jul 2020

Academic Editors: Su Pin Choo, Guang-Wen Cao Copy Editor: Cai-Hong Wang Production Editor: Tian Zhang

\begin{abstract}
Surgical resection or radiofrequency ablation (RFA) is considered first-choice treatment for small hepatocellular carcinomas (HCCs). When a patient has a small HCC that is inoperable or unsuitable for RFA, what are alternative treatments? Some oncologists recommend transarterial chemoembolization (TACE), chemotherapy, moleculartargeted therapy, or immunotherapy. However, these treatments have minimally beneficial effects in small HCCs. Stereotactic body radiation therapy (SBRT) is a liver-directed radical therapy for small HCCs, with treatment outcomes similar to those for surgical resection or RFA, but many oncologists do not comprehend its efficacy or accept this therapy. We herein discuss 11 typical patients who received SBRT for various indications: refusal to undergo resection or RFA; surgical resection or RFA considered difficult or unfeasible; residual cancer after surgical resection or RFA or incomplete iodized oil retention after TACE; or tumor recurrence after resection or RFA. We describe each case, including the radiation field, tumor radiation dose, and response to SBRT in both the tumor and liver parenchyma. These clinical data should help readers understand this new therapeutic technique. We also conducted a literature review and found evidence to support survival benefit with SBRT, including good three- and five-year overall survival rates. The purpose of this article is to encourage readers to accept the concept that SBRT is a low-toxicity and effective therapeutic option for patients with small HCCs, which offers substantial local control and improved overall survival, especially for patients with a tumor that is unresectable or unsuitable for RFA, residual tumor after local therapy, or intrahepatic recurrent tumor.
\end{abstract}

Keywords: Small hepatocellular carcinomas, stereotactic body radiation therapy, treatment outcomes, toxicity, imaging changes

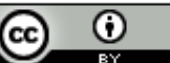

(C) The Author(s) 2020. Open Access This article is licensed under a Creative Commons Attribution 4.0 International License (https://creativecommons.org/licenses/by/4.0/), which permits unrestricted use, sharing, adaptation, distribution and reproduction in any medium or format, for any purpose, even commercially, as long as you give appropriate credit to the original author(s) and the source, provide a link to the Creative Commons license, and indicate if changes were made. 


\section{INTRODUCTION}

Recent technological advances offer precise and safe radiation delivery to tumors in various areas of the body through imaging guidance. External beam radiation therapy (EBRT) has been recommended as a therapeutic option for HCCs that are considered unresectable according to National Comprehensive Cancer Network guidelines because of the tumor's location, inadequate hepatic reserve, or the presence of comorbidities. SBRT is a type of EBRT technique requiring special equipment for patient positioning and the delivery of high-dose radiation to tumors. There is growing evidence supporting the usefulness of SBRT for patients considered unsuitable for hepatectomy or RFA. However, most oncologists or hepatologists do not understand this treatment technique because of limited use of SBRT in their clinical practice. Herein, we discuss 11 typical patients with HCC to clarify the indications, therapeutic outcomes, treatment-related toxicities, and doses of SBRT, as well as the expected post-SBRT imaging features.

\section{DEFINITIONS}

SBRT is an advanced technique of hypofractionated EBRT with photons, which delivers large ablative doses of radiation to tumors. This complex technique relies on the following: (1) stringent control of breathing motion for liver cancer, using four-dimensional computed tomography scans to track respiration-induced hepatic movement; (2) extremely precise patient positioning; and (3) image guidance for radiation delivery.

Early-stage HCC is defined as a solitary tumor with a maximum diameter $\leq 5 \mathrm{~cm}$ or multiple nodules ( $\leq 3$ total), each with a maximum diameter $\leq 3 \mathrm{~cm}$, without vascular invasion or extrahepatic metastasis and accompanied by Child-Pugh Class A or B hepatic function (Cases 1-3 and 5). Not all small tumors are classified as early-stage because some have decreased in size after treatment (Case 8). If intrahepatic recurrence (Cases 9 and 10) or Child-Pugh Class C (Case 11) function is present, HCC is considered later stage.

\section{CLINICAL EFFECTIVENESS OF SBRT FOR HCC}

There is a growing body of evidence indicating the usefulness of SBRT for the management of patients with HCC. We conducted a literature review and identified several retrospective studies involving the use of SBRT for HCC. These studies have primarily included patients in whom surgical resection or RFA was difficult, unfeasible, or rejected, as well as some patients with intermediate- or advanced-stage HCC. The results of these studies are summarized in Table 1 . This table is restricted to studies involving the use of $\leq 10$ fractions of SBRT.

\section{Overall survival}

As shown in Table 1, overall survival (OS) rates have varied between studies. For early-stage HCC, 2-year OS rates of $78 \%-80 \%{ }^{[1,2]}, 3$-year OS rates of $66 \%-73 \%^{[2,3]}$, and a 5 -year OS rate of $64 \%$ after SBRT have been reported $^{[3]}$.

\section{Local tumor control}

As shown in Table 1, local tumor control rates at one and two years were approximately $95 \%$ in most studies, especially those reported in more recent years ${ }^{[1-13]}$.

\section{Bridging before liver transplantation}

SBRT is suitable bridging therapy for patients with HCC awaiting liver transplantation. Sapisochin et al. ${ }^{[14]}$ compared the efficacy of SBRT, TACE, and RFA as a bridge to transplantation in a large cohort of patients with HCC and concluded that SBRT can be a safe alternative to the other, more conventional bridging therapies. However, SBRT has been safer than RFA and TACE when ascites or poor coagulation function are present, as often occurs in patients with underlying liver disease. 
Table 1. Outcomes of small-sized liver cancers after SBRT

\begin{tabular}{|c|c|c|c|c|c|c|c|c|c|c|c|c|c|c|c|}
\hline \multirow{2}{*}{ Authors } & \multirow{2}{*}{ Year } & \multirow{2}{*}{ HCC status } & \multirow{2}{*}{ Cases } & \multirow{2}{*}{ Dose to tumors } & \multicolumn{3}{|c|}{ Response(\%) } & \multicolumn{5}{|c|}{ Overall survival(\%) } & \multicolumn{3}{|c|}{ Local control (\%) } \\
\hline & & & & & CR & PR & SD & PD & $1-Y$ & $2-Y$ & 3-Y & $5-Y$ & $1-Y$ & $2-Y$ & $3-Y$ \\
\hline \multirow[t]{2}{*}{ Kimura et al. ${ }^{[1]}$} & \multirow[t]{2}{*}{2018} & \multirow{2}{*}{$\begin{array}{l}\text { BCLC } 053.3 \% \text {; BCLC } \\
\text { A } 46.7 \% \text {, Inoperable or } \\
\text { unsuitable RFA, or refusal } \\
\text { operation or RFA }\end{array}$} & 28 & $48 \mathrm{~Gy} / 4 \mathrm{Fx}$ & & & & & 78.6 & & & & 95.4 & 95.4 & \\
\hline & & & 122 & TACE + 48Gy/4Fx & & & & & 80.3 & & & & 99.2 & 98.5 & \\
\hline Takeda et al. ${ }^{[2]}$ & 2016 & $\begin{array}{l}\text { BCLC 0-A } 84 \%, \text { C } 16 \% \text {, } \\
\text { Salvage SBRT } 42 \% \text {, initial } \\
\text { SBRT } 36 \% \text {, intrahepatic } \\
\text { recurrence } 22 \%\end{array}$ & 90 & $\begin{array}{l}35 \mathrm{~Gy} / 5 \mathrm{Fx}(10 \%) \\
40 \mathrm{~Gy} / 5 \mathrm{Fx}(90 \%)\end{array}$ & & & & & 95.5 & 80.0 & 66.7 & & 98.8 & 96.3 & 96.3 \\
\hline Su et al. ${ }^{[3]}$ & 2016 & $\begin{array}{l}\text { Max. } \varnothing \leq 5 \mathrm{~cm} ; \text { BCLC } \\
\text { stage A } 55.3 \% \text {, B } 44.7 \% \\
\text { CP A } 86.4 \%, \text { CP B } 13.6 \%\end{array}$ & 132 & $42-46 G y / 3-5 F x$ & & & & & 94.1 & & 73.5 & 64.3 & 90.9 & & \\
\hline Wahl et al. ${ }^{[4]}$ & 2016 & $\begin{array}{l}\operatorname{Max} \varnothing<3 \mathrm{~cm} 73.1 \% ; 3 \\
\mathrm{~cm} \leq \varnothing<5 \mathrm{~cm} \mathrm{23.2 \% ;} \varnothing \\
\geq 5 \mathrm{~cm} 3.7 \%, \text { CP A } 68.7 \% \\
\text { B } 28.9 \%, \text { C } 2.4 \%\end{array}$ & 63 & $\begin{array}{l}30 \mathrm{~Gy} / 3 \mathrm{Fx}- \\
50 \mathrm{~Gy} / 5 \mathrm{Fx}\end{array}$ & & & & & 74 & 46 & & & 97.4 & 83.8 & \\
\hline Huertas et al. ${ }^{[5]}$ & 2015 & $\begin{array}{l}\varnothing \leq 6 \mathrm{~cm}, \text { CP: } \text { A5-B8, } \\
\text { ECOG } \leq 2, \text { nodules } \leq 3 \\
\text { AJCC stage I } 28.6 \%, \text { II } \\
68.8 \%, \text { IIIa } 1.3 \%, \text { IIIb } 1.3 \%\end{array}$ & 77 & $45 \mathrm{~Gy} / 3 \mathrm{Fx}, 2 \mathrm{Fx} / \mathrm{W}$ & & & & & 81.8 & 56.6 & & & 99 & 99 & \\
\hline Yamashita et al. ${ }^{[6]}$ & 2014 & $\begin{array}{l}\text { AJCC stage } 137 \% \text {, stage } \\
\text { II } 27 \% \text {, stage III } 8 \% \text {, } \\
\text { recurrence } 14 \% \text {, no stage: } \\
14 \%\end{array}$ & 79 & $\begin{array}{l}\text { BED10 }=96.3 \mathrm{~Gy} \\
(75-106) \\
\text { 40Gy/4Fx- } \\
60 \mathrm{~Gy} / 10 \mathrm{Fx}\end{array}$ & 45.6 & 35.4 & 11.4 & 5.1 & & 52.9 & & & & 74.8 & \\
\hline Lo et $a l_{.}^{[7]}$ & 2014 & $\begin{array}{l}\text { BCLC A 5.7\%; B 11.3\%; C } \\
83.0 \%\end{array}$ & 53 & $40 G y / 4-5 F x$ & 32.8 & 38.8 & 23.9 & 4.5 & 70.1 & 45.4 & & & 73.3 & 66.8 & \\
\hline Sanuki et al. ${ }^{[8]}$ & 2014 & $\begin{array}{l}\leq 5 \mathrm{~cm} . \text { T1 84.3\%; T2 } \\
11.4 \% ; \text { T3 4.3\% }\end{array}$ & 185 & $\begin{array}{l}\text { CP A: 40Gy/5Fx } \\
\text { CP B:35Gy/5Fx }\end{array}$ & & & & & 95 & 83 & 70 & & 99 & 93 & 91 \\
\hline Tekeda et al. ${ }^{[9]}$ & 2014 & $\begin{array}{l}\text { T1: 68.3\%, T2: 15.9\%, } \\
\text { T3:15.8\% }\end{array}$ & 63 & $35-40 G y / 5 F x$ & 80.7 & 17.7 & 1.6 & 0 & 100 & 87 & 73 & & 100 & 95 & 92 \\
\hline Yoon et al. ${ }^{[10]}$ & 2013 & $\begin{array}{l}\varnothing<6 \mathrm{~cm} ; \leq 3 \text { nodules; } \\
\mathrm{CP} \mathrm{A} \text { or } \mathrm{B} \text {; normal liver } \\
\text { volume }>700 \mathrm{~mL} \text {; } \\
\text { distance between tumor } \\
\text { and } \mathrm{GI}>2 \mathrm{~cm} \text {; } 92 \text { pts pre- } \\
\text { treatment failure }\end{array}$ & 93 & $30-60 G y / 3 F x$ & 51.5 & 21.4 & 25.2 & 0 & 86 & 53.8 & & & 94.8 & & 92.1 \\
\hline Jang et al. ${ }^{[1]]}$ & 2013 & $\begin{array}{l}\text { BCLC A: } 53 \% ; B: 29 \% ; C: \\
18 \% . \varnothing<7 \mathrm{~cm}\end{array}$ & 82 & $33-60 G y / 3 F x$ & & & & & & 63 & & 39 & & 87 & \\
\hline Bibault et al. ${ }^{[12]}$ & 2013 & $\begin{array}{l}\text { BCLC A: } 62.7 \% \text {; B: } 13.3 \% \text {; } \\
\text { C: } 24 \% .51 \% \text { treated with } \\
\text { other therapies }\end{array}$ & 75 & $\begin{array}{l}24-45 G y / 3 F x \\
\text { (median } 45 \text { Gy) }\end{array}$ & & & & & 78.5 & 50.4 & & & 89.8 & 89.8 & \\
\hline Park et al. ${ }^{[13]}$ & 2013 & $\begin{array}{l}\varnothing<6 \mathrm{~cm} \text {; nodules } \leq 3 \\
\text { normal liver volume }>700 \\
\mathrm{~mL} \text {; tumor between } \mathrm{Gl}>2 \\
\mathrm{~cm}\end{array}$ & 26 & $40-50 G y / 4-5 F x$ & 25 & 42.9 & 32.1 & 0 & 88.5 & 67.2 & & & & 87.6 & \\
\hline
\end{tabular}

SBRT: stereotactic body radiation therapy; HCC: hepatocellular carcinoma; CR: complete response; PR: partial response; SD: stable disease; PD: progressive disease; BCLC: Barcelona Clinic Liver Cancer; RFA: radiofrequency ablation; TACE: transarterial chemoembolization; CP: Child-Pugh Classification; Ø: diameter; ECOG: Eastern Cooperative Oncology Group; AJCC: American Joint Committee on Cancer; BED: biologically equivalent dose; GI: gastrointestine

\section{RADIATION DOSE}

The SBRT dose to HCC has been significantly associated with OS $(P=0.005)$ in multivariate analysis. Highdose SBRT may increase local control and improve OS in patients with inoperable $\mathrm{HCC}^{[11]}$. In most studies described in the literature, a biologically-equivalent dose (BED) 10 of $>80$ Gy has been delivered to the tumors [Table 1]. Of the 11 typical patients discussed in this manuscript, all received BED10 of $\geq 78$ Gy and are alive and well. Although no evidence has emerged to clearly support a minimum or maximum dose of SBRT for HCC, we recommend BED 10 of $\geq 80$ Gy.

\section{INDICATIONS FOR SBRT}

SBRT may be an effective therapeutic option for early-stage HCC (as defined above). Patients with earlystage HCC usually undergo surgical resection or RFA attempts, unless contraindicated. However, SBRT can 

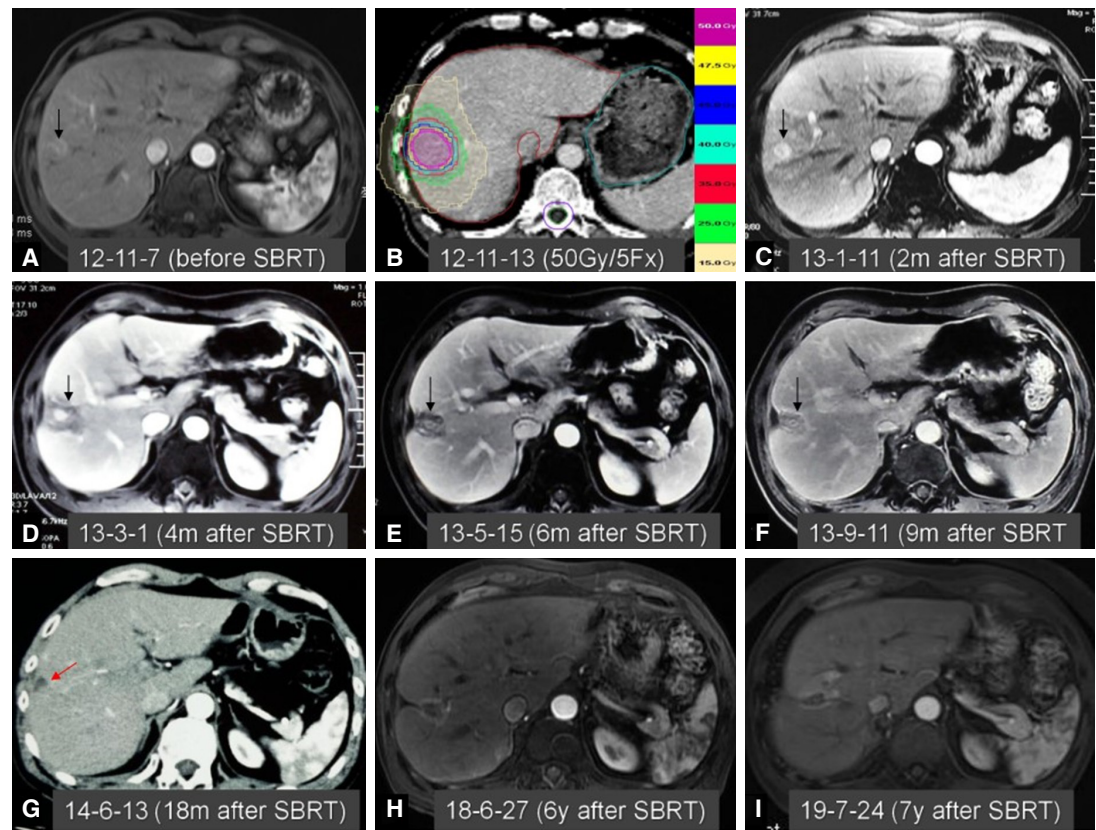

Figure 1. SBRT for HCC in a 59-year-old man who refused surgical resection and RFA. A: Axial arterial phase MRI image showed hyperenhancement of a $1.8-\mathrm{cm}$ liver nodule in the right lobe of the liver; B: the patient received SBRT with a dose of 50 Gy in five fractions; C: axial arterial phase MRI image 2 months after SBRT revealed a stable-size, enhancing lesion (arrowhead). The normal liver parenchyma received $25 \mathrm{~Gy}$ and appeared as a low-density area, indicating acute radiation injury; D: axial arterial phase MRI image 4 months after SBRT showed decreased size of the tumor, with atrophy of the perilesional hepatic parenchyma; E: axial arterial phase MRI image 6 months after SBRT showed necrotic changes and a hypovascular target lesion. The perilesional hepatic parenchyma, which received high-dose radiation, also exhibited necrotic changes; F: axial arterial phase MRI image nine months after SBRT demonstrated complete regression of the treated lesion and atrophy of the irradiated hepatic parenchyma; G: axial arterial phase CT image 18 months after SBRT showed a scar in the radiation field. Complete repair of the radiation damage was also observed; $\mathrm{H}, \mathrm{l}$ : axial arterial phase MRI images 6 years $(\mathrm{H})$ and 7 years $(\mathrm{I})$ after SBRT demonstrated a stable lesion size but the presence of complete necrosis and ring enhancement, indicating a complete radiologic response. Note the SBRT-related changes, which can be differentiated from tumor recurrence. SBRT: stereotactic body radiation therapy; HCC: hepatocellular carcinoma; RFA: radiofrequency ablation; MRI: magnetic resonance imaging; CT: computed tomography

be a useful alternative treatment in patients who refuse surgery or RFA. Case 1 [Figure 1] is a typical example of this.

Currently, SBRT is most frequently used when surgical resection or RFA would be difficult or unfeasible, as with tumors located at the center of liver, in the hepatic hilar region, or close to large blood vessels (as in Cases 2 and 3, which are shown in Figures 2 and 3). It is also commonly used in patients who are older [Case 4; Figure 4] or have significant comorbidities, such as poor liver function [Case 5; Figure 5].

SBRT may also be used for residual cancer after surgical resection, as in Case 6 [Figure 6], or after RFA, as in Case 7 [Figure 7]. In these situations, the tumor is often expected to be difficult to treat with surgery or RFA, but these treatments are tried initially. When residual tumor is found during follow-up, SBRT will generally be the most appropriate treatment.

SBRT may serve as adjuvant treatment for intrahepatic tumors with incomplete iodized oil retention, as in Case 8 [Figure 8]. TACE can be used to reduce the size of HCCs; however, it is often considered palliative therapy because of incomplete iodized oil retention by intrahepatic tumors. Adjuvant SBRT can function as consolidation treatment, converting palliative therapy to potentially curative therapy.

SBRT has also been used as salvage treatment for intrahepatic tumor recurrence after RFA or surgical resection [Cases 9 and 10; Figures 9 and 10]. Although metachronous intrahepatic recurrence is sometimes 

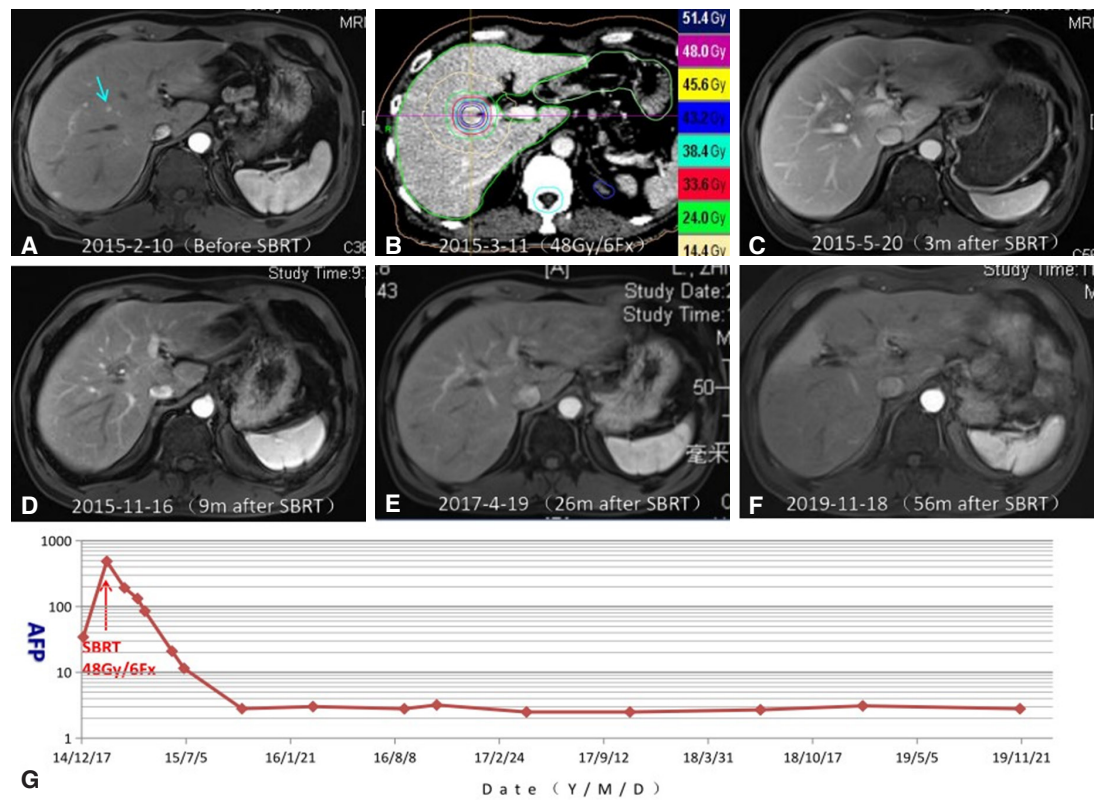

Figure 2. SBRT for very early-stage HCC in a 52-year-old man. Both surgical resection and RFA were considered difficult because the tumor was located in the center of the liver and was too small to be easily detected. A: Axial arterial phase MRI image showed hyper enhancement of an $0.8-\mathrm{cm}$ liver nodule (arrowhead) located in the center of the right lobe of the liver. The patient was clinically diagnosed with very early-stage HCC based on the Barcelona Clinic Liver Cancer staging system; B: the patient received SBRT with a dose of $48 \mathrm{~Gy}$ in six fractions; C: axial arterial phase MRI image three months after SBRT demonstrated complete tumor response. Hypodensity in the radiation field (about $30 \mathrm{~Gy}$ ) indicated the presence of radiation-induced focal liver injury; D: axial arterial phase MRI image 9 months after SBRT showed clear reduction in size of the area of radiation injury; E,F: axial arterial phase MRI images 26 months (E) and 56 months (F) after SBRT revealed complete regression of the tumor lesion; G: serum AFP levels are shown in relation to the treatment timeline. The elevated serum AFP level prior to SBRT dramatically declined to normal $(<20 \mu \mathrm{g} / \mathrm{L})$ after SBRT, and remained within normal limits thereafter. SBRT: stereotactic body radiation therapy; HCC: hepatocellular carcinoma; RFA: radiofrequency ablation; MRI: magnetic resonance imaging; AFP: alpha-fetoprotein
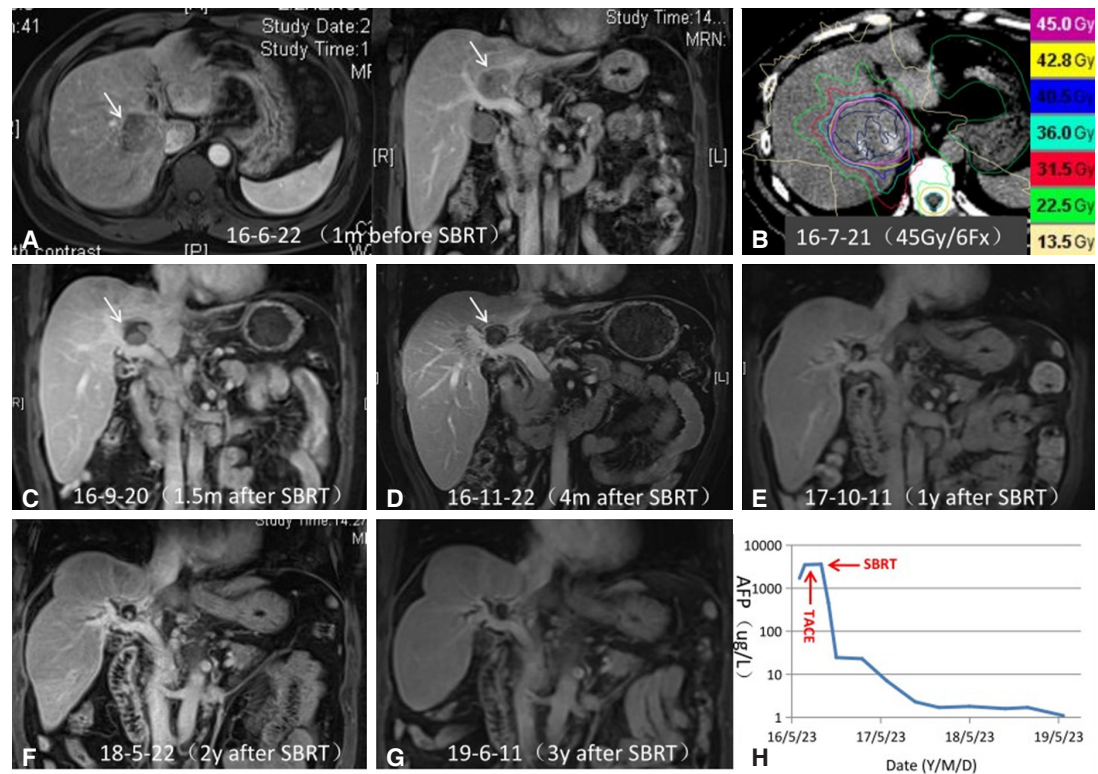

Figure 3. SBRT for unresectable HCC in a 47-year-old man. A: Axial and sagittal MRI images showed a hepatic lesion (arrowhead) near the inferior vena cava and main portal vein. The lesion enhanced in the arterial phase and washed out in the portal venous phase; $\mathrm{B}$ : the patient underwent SBRT with a dose of $45 \mathrm{~Gy}$ in six fractions; C: arterial phase MRI image 1.5 months after SBRT revealed dramatic regression of the lesion; D: arterial phase MRI image four months after SBRT demonstrated further reduction in size of the lesion, as well as necrosis of the targeted hypovascular lesion, consistent with a nonviable tumor; E-G: MRI images 1 year (E), 2 years (F), and 3 years (G) after SBRT showed progressive reduction in tumor size and complete hypovascularity of the lesion. These findings suggested a good tumor response; $\mathrm{H}$ : serum AFP levels are shown in relation to the treatment timeline. The elevated serum AFP level prior to SBRT $(1,709 \mu \mathrm{g} / \mathrm{L})$ declined to normal $(<20 \mu \mathrm{g} / \mathrm{L})$ after SBRT and remained within normal limits thereafter. SBRT: stereotactic body radiation therapy; HCC: hepatocellular carcinoma; MRI: magnetic resonance imaging; AFP: alpha-fetoprotein 

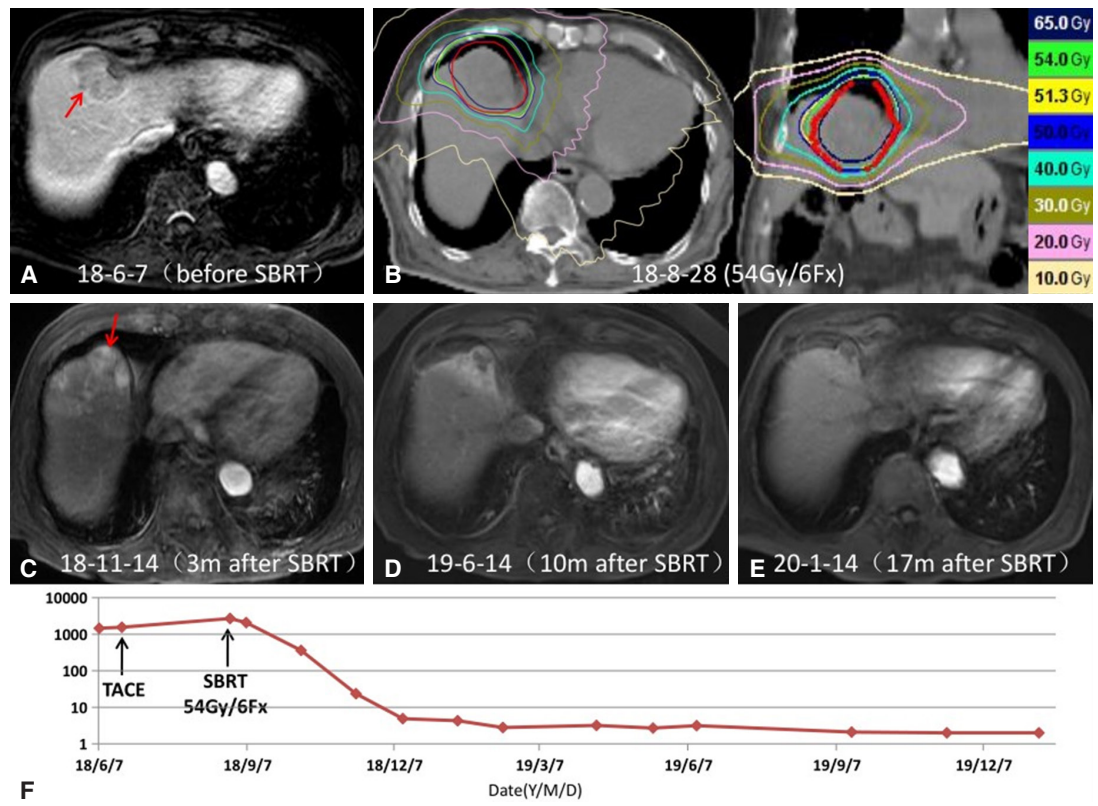

Figure 4. SBRT for HCC in a 99-year-old man for whom SBRT was chosen because of his age and comorbidities. A: Axial venous phase MRI image showed a 6-cm low-density lesion adjacent to the diaphragm (arrow); B: the patient underwent SBRT with a dose of 54 Gy in six fractions; C: axial arterial phase MRI image three months after SBRT demonstrated reduced size of the intrahepatic lesion and vascular enhancement (arrow); D,E: axial arterial phase MRI images 10 months (D) and 17 months (E) after SBRT showed further reduction in size of the tumor, which was hypovascular (arrows). The perilesional liver parenchyma in the enhanced phase appeared as ill-defined areas of enhancement, favoring SBRT-related changes; F: Serum AFP levels are shown in relation to the treatment timeline. AFP levels decreased to normal after SBRT. SBRT: stereotactic body radiation therapy; HCC: hepatocellular carcinoma; MRI: magnetic resonance imaging; AFP: alpha-fetoprotein
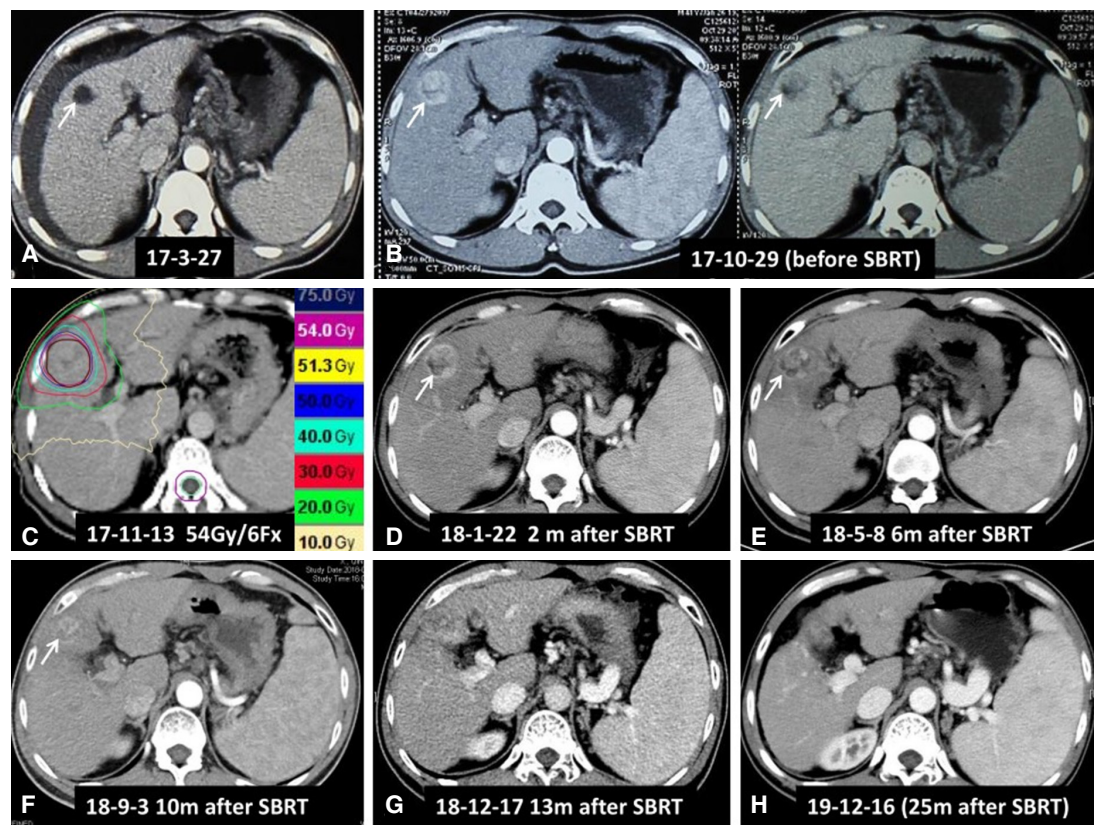

Figure 5. SBRT for $\mathrm{HCC}$ consisting of an extensive fat-tissue component in a 41-year-old man with Child-Pugh Class $\mathrm{C}$ liver function. A: Axial arterial phase CT image showed a 2-cm nodule containing fat attenuation (arrow). Mild ascites and splenomegaly were also evident; B: multiphasic CT images six months after liver protecting treatment demonstrated enlargement of the lesion to $3 \mathrm{~cm}$ (arrow), with enhancement in the arterial phase and washout in the portal venous phase. The patient was diagnosed with $\mathrm{HCC}$ with an extensive fat-tissue component. The ascites had disappeared, but splenomegaly was still present; C: the patient underwent SBRT with a dose of 54 Gy in six fractions; D: axial arterial phase CT image two months after SBRT showed the lesion (arrow) with a stable size and partial devascularization; E-G: axial arterial phase CT images 6 months (E), 9 months (F), and 13 months (G) after SBRT demonstrated progressive reduction in tumor size and devascularization of the lesion, consistent with a partial response based on $\mathrm{mRECIST}$ criteria; $\mathrm{H}$ : axial arterial phase CT image 25 months after SBRT showed complete response of the treated lesion, with marked focal atrophy of the irradiated hepatic parenchyma. SBRT: stereotactic body radiation therapy; HCC: hepatocellular carcinoma; CT: computed tomography; mRECIST: modified Response Evaluation Criteria In Solid Tumor 

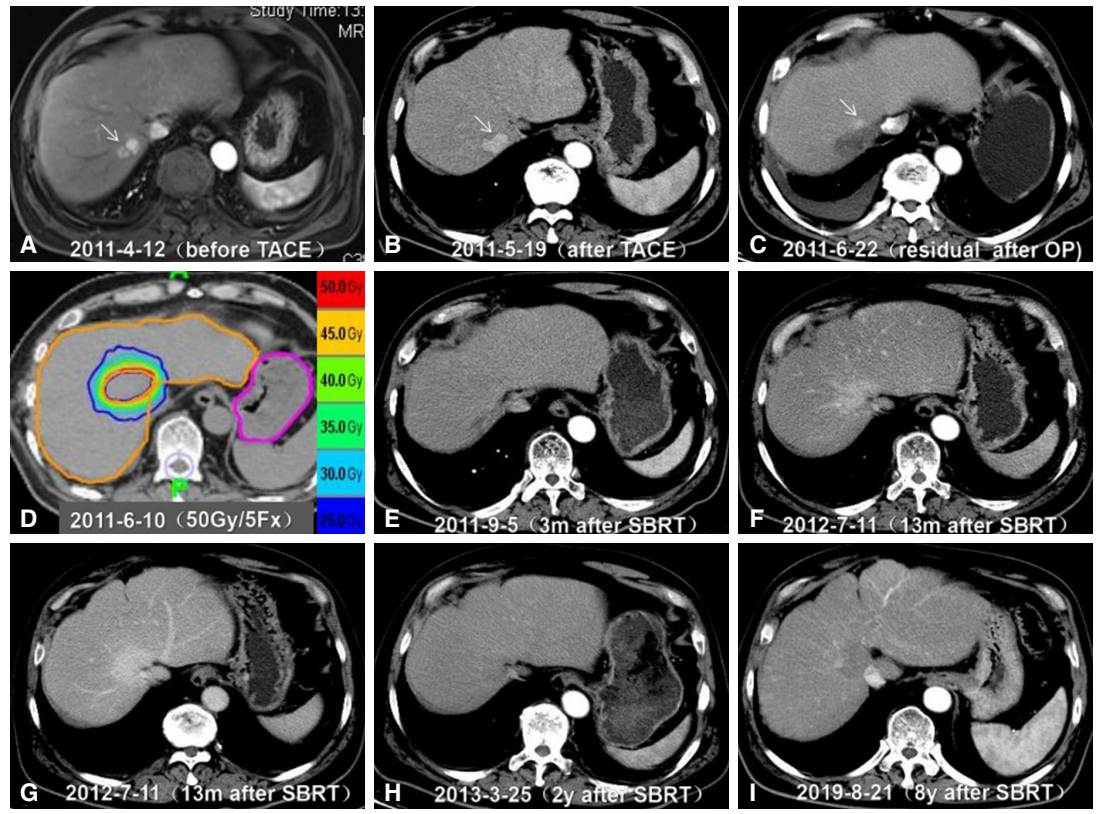

Figure 6. SBRT for residual HCC after surgical resection in a 59-year-old man. A: Axial arterial phase MRI scan 6 years after initial surgery showed a $2.1-\mathrm{cm}$ recurrent hyper-enhancing nodule adjacent to the inferior vein cava, located in the second hepatic hilar segment. The patient underwent TACE for the recurrent lesion; B: axial arterial phase CT image one month after TACE showed a hyper-enhancing lesion (arrow), with no lipiodol deposition. Then, the patient underwent hepatic wedge resection in May 2011; C: axial arterial phase CT image one month after surgery revealed residual tumor with hyper enhancement (arrow); D: the patient underwent SBRT with a dose of 50 Gy in five fractions in June 2011; E: axial arterial phase CT image 3 months after SBRT showed complete response of the target lesion; F,G: Axial arterial (F) and portal venous phases (G) CT images 13 months after SBRT showed persistent enhancement of the radiation field because of post-SBRT changes representing congestion and edema; $\mathrm{H}, \mathrm{l}$ : Follow-up CT images 2 years $(\mathrm{H})$ and 8 years $(\mathrm{I})$ after SBRT demonstrated a normal liver. SBRT: stereotactic body radiation therapy; HCC: hepatocellular carcinoma; MRI: magnetic resonance imaging; TACE: transarterial chemoembolization; CT: computed tomography
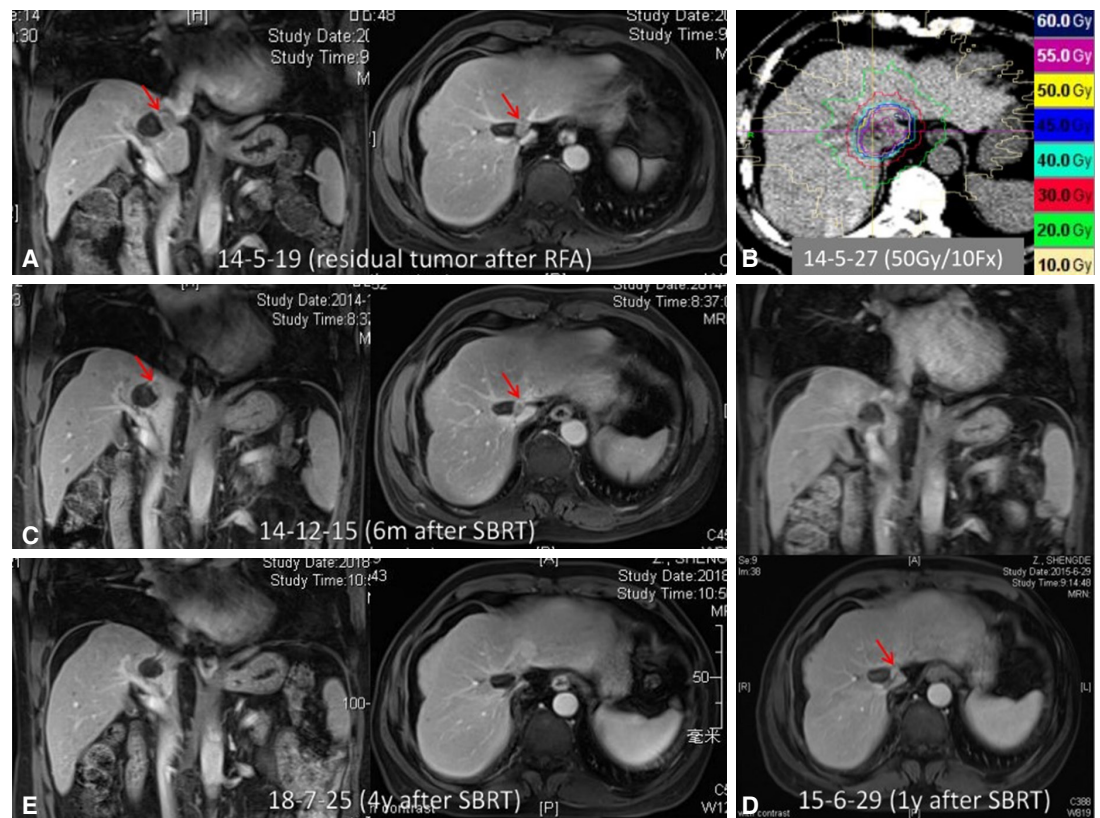

Figure 7. SBRT for residual tumor after RFA in a 66-year-old man. A: Axial and sagittal MRI images approximately 1.5 months after RFA showed a hepatic lesion with a residual cavity and viable tumor (arrowhead) close to the inferior vena cava; B: the patient underwent SBRT with a dose of $50 \mathrm{~Gy}$ in 10 fractions for the residual tumor; C-E: MRI images obtained 6 months (C), 1 year (D), and 4 years (E) after SBRT illustrate gradual shrinkage and eventual disappearance of the tumor. SBRT: stereotactic body radiation therapy; RFA: radiofrequency ablation; MRI: magnetic resonance imaging 

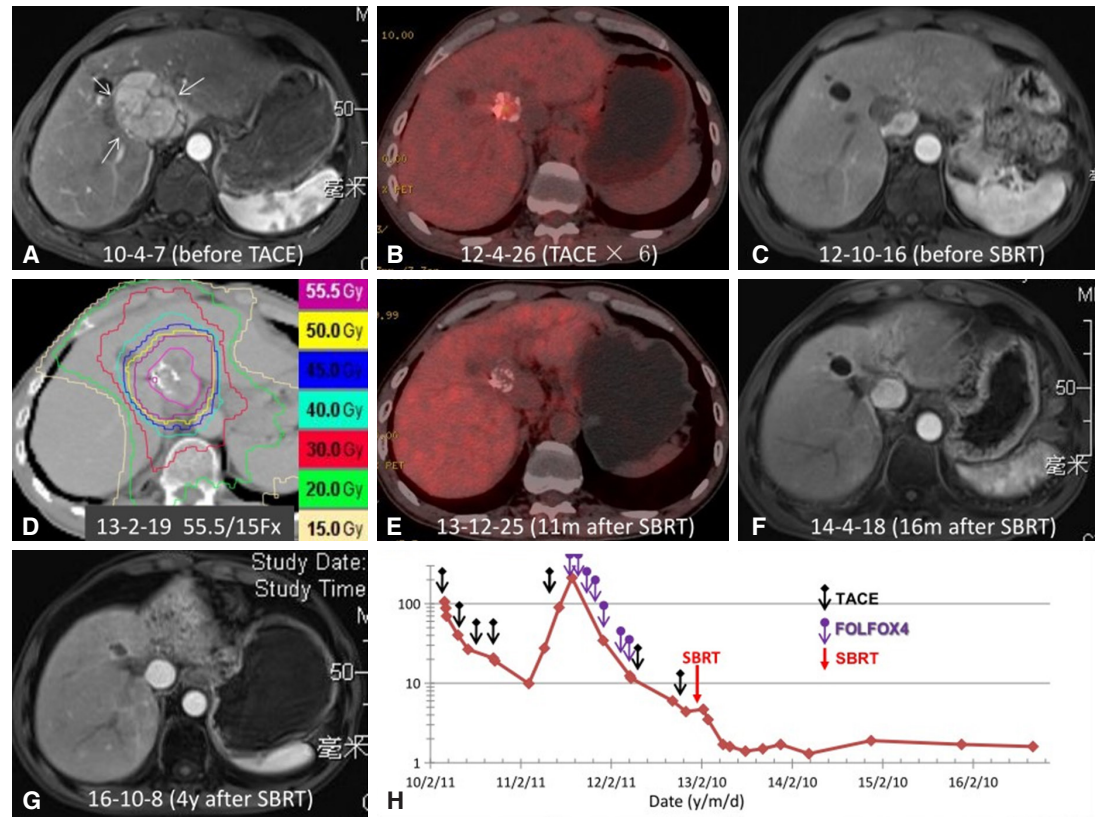

Figure 8. SBRT for residual tumor with incomplete iodized oil retention in a 54-year-old man who had undergone multiple cycles of TACE and chemotherapy. A: Original axial arterial phase MRI image showed a 7.5- $\mathrm{cm}$ enhancing mass in the hepatic hilar region, consistent with HCC; B: the lesion decreased substantially in size after six cycles of TACE. FDG PET/CT image demonstrated hypermetabolic activity in the region with incomplete iodized oil retention, consistent with residual viable tumor; C: arterial phase MRI image after multiple TACE cycles and chemotherapy but before SBRT showed no enhancement of the 1.8- $\mathrm{cm}$ liver lesion; D: the patient underwent SBRT for residual tumor with a dose of 55.5 Gy in 15 fractions; E: FDG PET/CT image 11 months after SBRT showed no metabolic activity; F: axial arterial phase MRI image 16 months after SBRT demonstrated reduced size and hypovascularity of the treated lesion; G: axial arterial phase MR image 4 years after SBRT showed stable size and hypovascularity of the treated lesion; H: serum AFP levels are shown in relation to the treatment timeline. AFP further declined to normal after SBRT. SBRT: stereotactic body radiation therapy; HCC: hepatocellular carcinoma; MRI: magnetic resonance imaging; TACE: transarterial chemoembolization; AFP: alpha-fetoprotein; FDG: flurodeoxyglucose; PET: positron emission tomography; CT: computed tomography
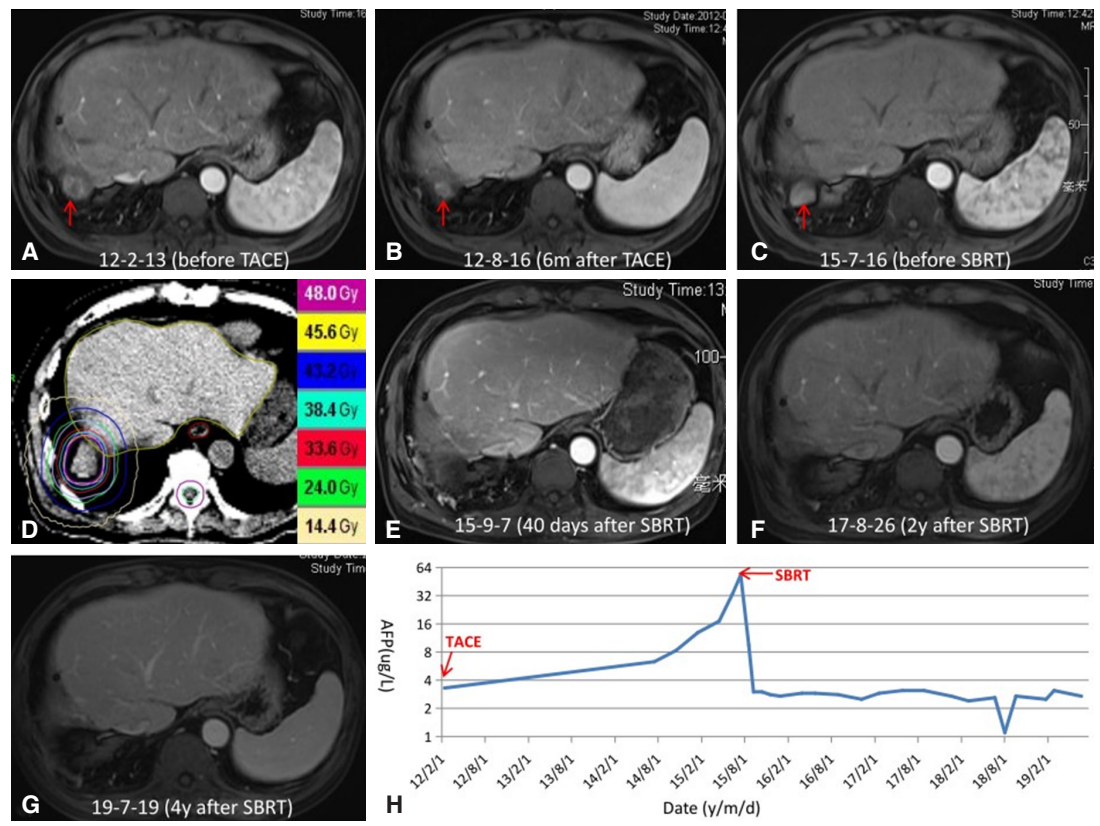

Figure 9. SBRT for recurrent $\mathrm{HCC}$ at the surgical margin in a 63-year-old man. A: Axial arterial phase MRI image showed hyper enhancement of a $2.4 \mathrm{~cm}$ recurrent focus at the surgical margin (arrowhead). The patient received TACE for this recurrent lesion; B: axial arterial phase MRI image six months after TACE showed reduced size and hypovascularity of the lesion; $\mathrm{C}$ : axial arterial phase CT image 3 years after TACE demonstrated enlargement of the treated lesion to $2.9 \mathrm{~cm}$; D: the patient received SBRT with a dose of $48 \mathrm{~Gy}$ in six fractions; E-G: CT images 40 days (E), 2 years (F), and 4 years (G) after SBRT showed complete tumor response; H: serum AFP levels are shown in relation to the treatment timeline. The elevated serum AFP prior to SBRT $(53 \mu \mathrm{g} / \mathrm{L})$ declined to normal $(2.1 \mu \mathrm{g} / \mathrm{L})$ after SBRT and remained within normal limits thereafter. SBRT: stereotactic body radiation therapy; HCC: hepatocellular carcinoma; MRI: magnetic resonance imaging; TACE: transarterial chemoembolization; CT: computed tomography; AFP: alpha-fetoprotein 

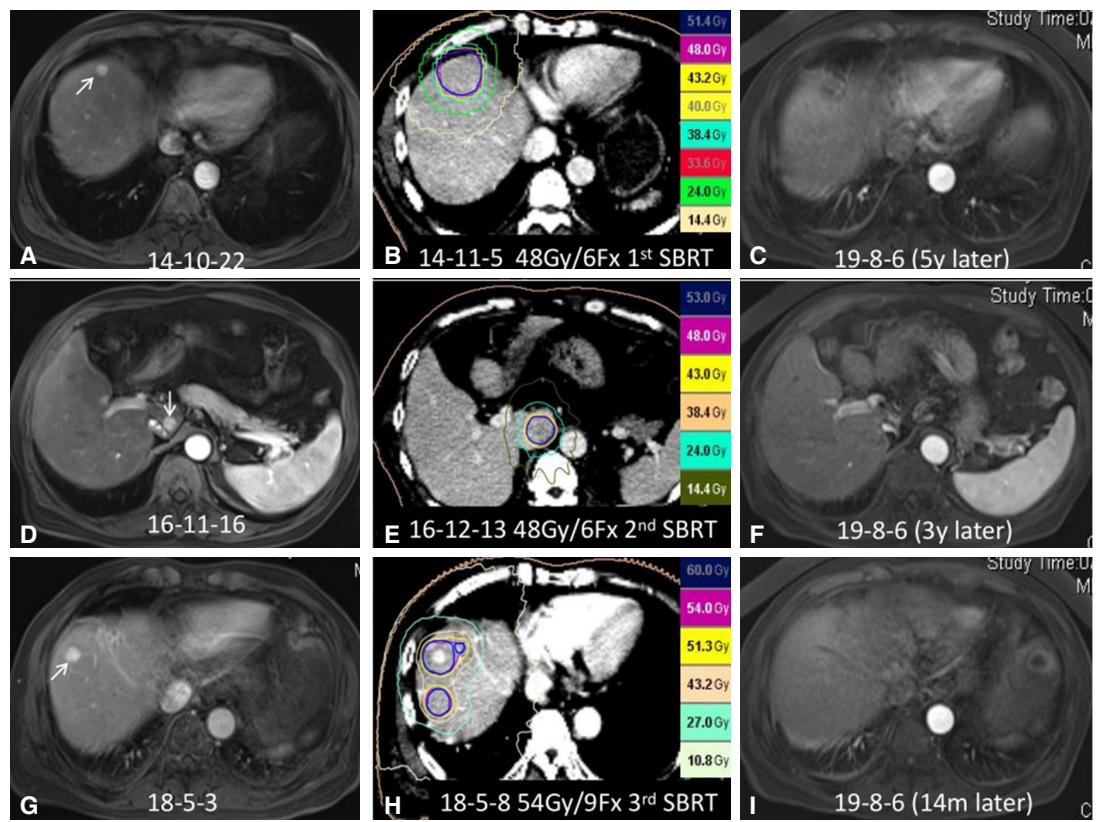

Figure 10. SBRT for repeated de novo HCC in a 63-year-old man with previous surgical resection of HCC. A: Axial arterial phase MRI image showed a 1.2-cm enhancing nodule in the right hepatic lobe, adjacent to the diaphragm; B: the patient received his first course of SBRT with a dose of 48 Gy in six fractions; C: axial arterial phase MRI image 4 years after SBRT demonstrated complete response of this right lobe lesion; D: axial arterial phase MRI image two years after the first SBRT showed a 1-cm enhancing nodule in the caudate lobe of the liver; E: the patient received a second course of SBRT with a dose of 48 Gy in six fractions; F: axial arterial phase MRI image 3 years after the second course of SBRT revealed complete response of the caudate lobe lesion; G: axial arterial phase MRI image 18 months after the second SBRT showed a 1.2-cm enhancing lesion in the right hepatic lobe, as well as an additional lesion at a lower level (not shown), suggesting de novo HCC; $\mathrm{H}$ : the patient received a third course of SBRT with a dose of $54 \mathrm{~Gy}$ in nine fractions. I: axial arterial phase MRI image one year after this SBRT revealed complete tumor response of the de novo lesion. SBRT: stereotactic body radiation therapy; HCC: hepatocellular carcinoma; MRI: magnetic resonance imaging
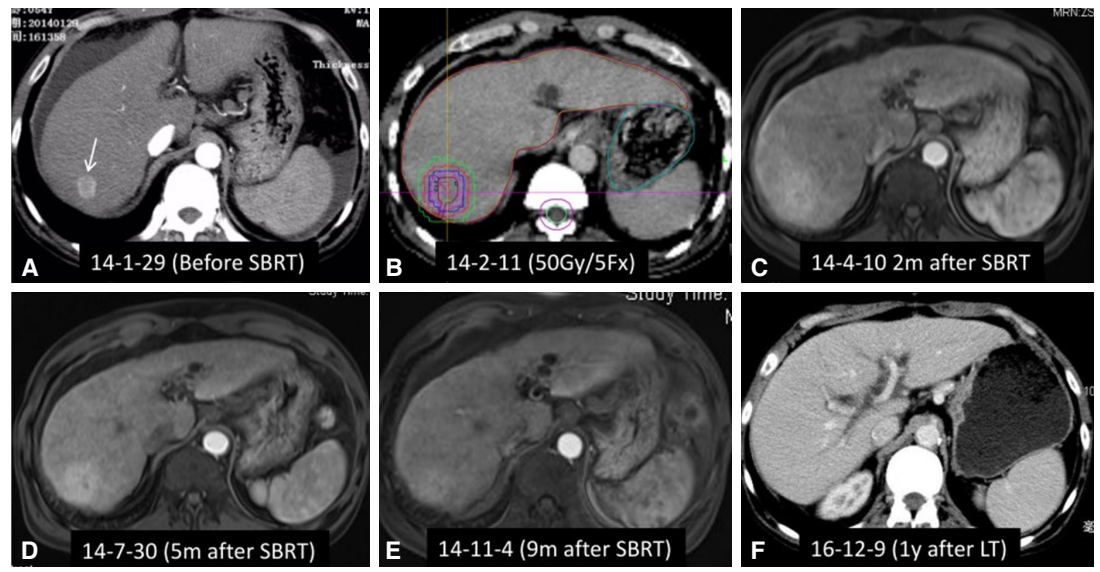

Figure 11. SBRT as a bridge to liver transplantation for HCC in a 55-year-old man with alcohol-related cirrhosis and Child-Pugh Class C liver function. A: Axial arterial phase CT image showed hyper enhancement of a $2 \mathrm{~cm}$ mass in the right hepatic lobe (arrowhead), accompanied by moderate ascites. Liver function was scored as Child-Pugh Class C; B: the patient underwent SBRT with a dose of 50 Gy in five fractions; C-E: Axial arterial phase MRI images 1.5 months (C), 5 months (D), and 9 months (E) after SBRT showed complete tumor response. However, focal reaction with enhancement indicated congestion of the hepatic parenchyma in the radiation field. The patient received a liver transplant 22 months after SBRT; F: axial arterial phase CT image one year after liver transplantation demonstrated a normal liver. SBRT: stereotactic body radiation therapy; HCC: hepatocellular carcinoma; MRI: magnetic resonance imaging; CT: computed tomography

difficult to distinguish from de novo HCC, a course of SBRT can be considered. In addition, SBRT can be a suitable bridging therapy for patients with HCC awaiting liver transplantation [Case 11; Figure 11]. 


\section{CONTRAINDICATIONS FOR SBRT IN SMALL HCC}

If tumors and luminal structures (esophagus, stomach, duodenum, or intestine) are closely situated at $<1 \mathrm{~cm}$, SBRT is relatively contraindicated for this patient. However, hypofraction imaging-guided radiation therapy could be recommended when HCC is inoperable or unsuitable for RFA.

At least $700 \mathrm{~mL}$ of normal liver (Child-Pugh Class A) must receive $<15$ Gy. If this condition is not met, we must be careful in choosing such patients.

The safety of liver radiation for HCC in patients with Child-Pugh Class C cirrhosis has not been established, but SBRT could be used as bridging therapy for patients with HCC awaiting liver transplantation.

\section{RESPONSE TO SBRT}

\section{$\mathrm{HCC}$}

Tumor response rates increase over time after SBRT. Sanuki et al. ${ }^{[15]}$ reported that HCC complete response increased from $24 \%$ at three months after SBRT to $67 \%, 71 \%$, and $93 \%$ at 6,12 , and 24 months, respectively, after SBRT ${ }^{[15]}$. Using modified Response Evaluation Criteria In Solid Tumor (mRECIST) criteria, complete response occurred within three months after completing SBRT in Cases 9 and 11 and $>9$ months after SBRT in Cases 1, 5, and 7. Of note, we prefer to evaluate tumor response to SBRT using European Association

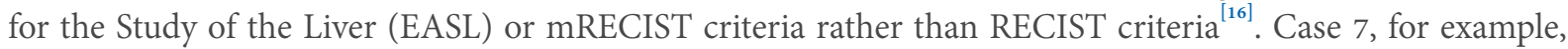
exhibited a complete response according to EASL or mRECIST criteria in the fourth year after completion of SBRT, but only a stable disease and partial response using RECIST criteria, at six months and one year after completing SBRT, respectively. Similarly, Case 5 had a partial response using EASL or mRECIST criteria, but stable disease using RECIST criteria, at six months after completing SBRT.

\section{Liver parenchymal reactions}

Early focal liver reaction refers to a surrounding low-intensity area observed on both computed tomography (CT) and magnetic resonance imaging (MRI) scans within six months after completing SBRT. This focal reaction is more visible in patients who undergo initial therapy with SBRT, as shown in Cases 1,2, and 5.

Delayed focal liver reactions are classified as areas of hyperdensity, isodensity, and hypodensity in all enhanced phases on follow-up MRI or CT $>6$ months after SBRT completion ${ }^{[17,18]}$. Features of hyperdensity were found in Cases 6 [Figure $6 \mathrm{~F}$ and $\mathrm{G}$ ] and 11 [Figure 11D]; features of isodensity were found in Cases 3 [Figure 3E], 7 [Figure 7C and D], and 11 [Figure 11E]; and features of hypodensity were found in Cases 1 [Figure 1E], 2 [Figure 2D], and 5 [Figure 5E].

The incidence of hyperdensity reactions in irradiated hepatic parenchyma may gradually increase after 6 months post-SBRT completion, potentially interfering with accurate assessment of treatment response and being misinterpreted as recurrent tumor. Lack of washout in the delayed phase in hypervascular areas helps distinguish SBRT-related changes from residual or recurrent $\mathrm{HCC}^{[19]}$. Hyperdensity will typically disappear 2-3 years after treatment, as shown in Case 6 [Figure 6H]. Hypodensity represents the presence of regional liver atrophy within 1-2 years, as shown in Cases 1 [Figure $1 \mathrm{~F}$ ], and 5 [Figure $5 \mathrm{H}$ ].

The types of focal reaction did not appear to be related to liver function in our cases. The focal liver reaction threshold dose following SBRT for HCC is 30 Gy for livers with Child-Pugh Class A function and 25 Gy for livers with Child-Pugh Class B function, when delivered in five fractions ${ }^{[20,21]}$. The doses used in our cases [Figures 1C, 2C, and 5D] were consistent with these thresholds.

\section{TOXICITY}

\section{Hepatic damage}

A consensus article summarizing the results of 15 previously published studies, including 1063 patients with HCC undergoing SBRT, reported that only eight patients (0.8\%) developed Grade 5 liver failure, and most 
fatalities from liver failure occurred in patients with Child-Pugh Class B liver function ${ }^{[22]}$. However, we have observed no fatal radiation-induced liver disease in our clinical practice.

The safety of SBRT for HCC in patients with Child-Pugh Class C liver function has not been established. Class $\mathrm{C}$ function is generally considered a contraindication to all HCC treatment except liver transplantation. No deterioration in liver function occurred after SBRT in Cases 5 and 11, who had Child-Pugh Class C liver function. Furthermore, some patients undergo $>1$ course of SBRT, as exemplified by Case 10, who received a total of three courses because of the development of de novo HCCs. Together, these observations suggest that SBRT produces little liver parenchymal damage.

\section{Other toxicities}

Gastrointestinal toxicity is another potential concern with SBRT, especially when luminal structures, such as the esophagus, stomach, duodenum, or intestines, are close to the tumors being treated. Grade 3 or higher gastrointestinal toxicities were reported in $1.4 \%$ of patients from previously published studies, but fatal gastrointestinal bleeding did not occur ${ }^{[22]}$. Other complications, such as rib fractures, chest or abdominal wall pain, biliary stricture, and musculoskeletal discomfort, have been noted occasionally. Overall, most toxicities from SBRT are generally infrequent and mild.

\section{THE LIMITATION OF SBRT}

Firstly, there is a lack of randomized clinical trials to compare the treatment outcome among SBRT, resection, and RFA. Thus, SBRT can only be used as an alternative treatment when operation and RFA are impossible. Secondly, vaguely defined SBRT has led to inconsistent radiation doses and fractions globally, with $\leq 5$ fractions in the United States and $\leq 10$ fractions in the rest of the world. A dose of 55.5 Gy in 15 fractions was delivered to Case 8, and complete response was achieved. This is not strictly part of SBRT; at this point, we are not interested in the definition of SBRT, as we care more about the local control and long-term survival.

\section{CONCLUSION}

SBRT is an effective therapeutic option based on proven studies for patients with small HCCs; is complementary to the existing treatment options, as illustrated by the typical cases; and is safe with minimal toxicities.

\section{DECLARATIONS}

\section{Authors' contributions}

Made a substantial, direct and intellectual contribution to the work, and approved it for publication: Zeng

ZC, Fan J, Zhou J, Zeng MS, Chen YX, Wu ZF, Sun J, Zhang JY, Hu Y, Zhao QQ

\section{Availability of data and materials}

Not applicable.

\section{Financial support and sponsorship}

The research was supported by a grant from the National Key R \& D Program of China (2017YFC0112100).

\section{Conflicts of interest}

All authors declared that there are no conflicts of interest.

\section{Ethical approval and consent to participate}

Not applicable.

\section{Consent for publication}

Not applicable. 


\section{Copyright}

(c) The Author(s) 2020.

\section{REFERENCES}

1. Kimura T, Aikata H, Doi Y, Kimura T, Aikata H, et al. Comparison of stereotactic body radiation therapy combined with or without transcatheter arterial chemoembolization for patients with small hepatocellular carcinoma ineligible for resection or ablation therapies Technol Cancer Res Treat 2018;17:1533033818783450.

2. Takeda A, Sanuki N, Tsurugai Y, Iwabuchi S, Matsunaga K, et al. Phase 2 study of stereotactic body radiotherapy and optional transarterial chemoembolization for solitary hepatocellular carcinoma not amenable to resection and radiofrequency ablation. Cancer 2016;122:2041-9.

3. Su TS, Liang P, Lu HZ, Liang J, Gao YC, et al. Stereotactic body radiation therapy for small primary or recurrent hepatocellular carcinoma in 132 Chinese patients. J Surg Oncol 2016;113:181-7.

4. Wahl DR, Stenmark MH, Tao Y, Pollom EL, Caoili EM, et al. Outcomes after stereotactic body radiotherapy or radiofrequency ablation for hepatocellular carcinoma. J Clin Oncol 2016;34:452-9.

5. Huertas A, Baumann AS, Saunier-Kubs F, Salleron J, Oldrini G, et al. Stereotactic body radiation therapy as an ablative treatment for inoperable hepatocellular carcinoma. Radiother Oncol 2015;115:211-6.

6. Yamashita H, Onishi H, Matsumoto Y, Murakami N, Matsuo Y, et al. Local effect of stereotactic body radiotherapy for primary and metastatic liver tumors in 130 Japanese patients. Radiat Oncol 2014;9:112.

7. Lo CH, Huang WY, Lee MS, Lin KT, Lin TP, et al. Stereotactic ablative radiotherapy for unresectable hepatocellular carcinoma patients who failed or were unsuitable for transarterial chemoembolization. Eur J Gastroenterol Hepatol 2014;26:345-52.

8. Sanuki N, Takeda A, Oku Y, Mizuno T, Aoki Y, et al. Stereotactic body radiotherapy for small hepatocellular carcinoma: a retrospective outcome analysis in 185 patients. Acta Oncol 2014;53:399-404.

9. Takeda A, Sanuki N, Eriguchi T, Kobayashi T, Iwabutchi S, et al. Stereotactic ablative body radiotherapy for previously untreated solitary hepatocellular carcinoma. J Gastroenterol Hepatol 2014;29:372-9.

10. Yoon SM, Lim YS, Park MJ, Kim SY, Cho B, et al. Stereotactic body radiation therapy as an alternative treatment for small hepatocellular carcinoma. PLoS One 2013;8:e79854.

11. Jang WI, Kim MS, Bae SH, Cho CK, Yoo HJ, et al. High-dose stereotactic body radiotherapy correlates increased local control and overall survival in patients with inoperable hepatocellular carcinoma. Radiat Oncol 2013;8:250.

12. Bibault JE, Dewas S, Vautravers-Dewas C, Hollebecque A, Jarraya H, et al. Stereotactic body radiation therapy for hepatocellular carcinoma: prognostic factors of local control, overall survival, and toxicity. PLoS One 2013;8:e77472.

13. Park JH, Yoon SM, Lim YS, Kim SY, Shim JH, et al. Two-week schedule of hypofractionated radiotherapy as a local salvage treatment for small hepatocellular carcinoma. J Gastroenterol Hepatol 2013;28:1638-42.

14. Sapisochin G, Barry A, Doherty M, Fischer S, Goldaracena N, et al. Stereotactic body radiotherapy vs. TACE or RFA as a bridge to transplant in patients with hepatocellular carcinoma. An intention-to-treat analysis. J Hepatol 2017;67:92-9.

15. Sanuki N, Takeda A, Mizuno T, Oku Y, Eriguchi T, et al. Tumor response on CT following hypofractionated stereotactic ablative body radiotherapy for small hypervascular hepatocellular carcinoma with cirrhosis. AJR Am J Roentgenol 2013;201:W812-20.

16. Oldrini G, Huertas A, Renard-Oldrini S, Taste-George H, Vogin G, et al. Tumor response assessment by MRI following stereotactic body radiation therapy for hepatocellular carcinoma. PLoS One 2017;12:e0176118.

17. Sanuki-Fujimoto N, Takeda A, Ohashi T, Taste-George H, Vogin G, et al. CT evaluations of focal liver reactions following stereotactic body radiotherapy for small hepatocellular carcinoma with cirrhosis: relationship between imaging appearance and baseline liver function. Br J Radiol 2010;83:1063-71.

18. Kimura T, Takahashi S, Takahashi I, Nishibuchi I, Doi Y, et al. The time course of dynamic computed tomographic appearance of radiation injury to the cirrhotic liver following stereotactic body radiation therapy for hepatocellular carcinoma. PLoS One 2015; 10:e125231.

19. Park MJ, Kim SY, Yoon SM, Kim JH, Park SH, et al. Stereotactic body radiotherapy-induced arterial hypervascularity of nontumorous hepatic parenchyma in patients with hepatocellular carcinoma: potential pitfalls in tumor response evaluation on multiphase computed tomography. PLoS One 2014;9:e90327.

20. Sanuki N, Takeda A, Oku Y, Eriguchi T, Nishimura S, et al. Threshold doses for focal liver reaction after stereotactic ablative body radiation therapy for small hepatocellular carcinoma depend on liver function: evaluation on magnetic resonance imaging with Gd-EOBDTPA. Int J Radiat Oncol Biol Phys 2014;88:306-11.

21. Takeda A, Oku Y, Sanuki N, Kunieda E, Koike N, et al. Dose volume histogram analysis of focal liver reaction in follow-up multiphasic CT following stereotactic body radiotherapy for small hepatocellular carcinoma. Radiother Oncol 2012;104:374-8

22. Zeng ZC, Seong J, Yoon SM, Cheng JC, Lam KO, et al. Consensus on stereotactic body radiation therapy for small-sized hepatocellular carcinoma at the 7th Asia-Pacific Primary Liver Cancer Expert Meeting. Liver Cancer 2017;6:264-74. 